\title{
Enhanced Dissolution of Coenzyme Q10 using Solid Dispersions Prepared by Low Temperature Melting Method
}

\author{
Jun Heok Kang ${ }^{1}$, Yi-Dong Yan ${ }^{1}$, Hyun Chan Kim ${ }^{1}$, Sung Neung Lee ${ }^{1}$, \\ Chul Soon Yong ${ }^{1 \dagger}$ and Han-Gon Choi ${ }^{1, \dagger}$ \\ ${ }^{1}$ College of Pharmacy, Yeungnam University, 214-1, Dae-Dong, Gyongsan 712-749, South Korea \\ ${ }^{2}$ College of Pharmacy, Hanyang University, 1271, Sa-3-Dong, Ansan 426-791, South Korea
}

(Received July 15, 2010 • Revised October 5, 2010 • Accepted October 6, 2010)

\begin{abstract}
CoQ with low melting temperature was exploited to improve its solubility by preparing its solid dispersions (SDs) with a meltable polymer, poloxamer 407 (P 407). P407 can be utilized for a relatively simple, quick, inexpensive, reproducible and potentially scalable manner in the low temperature melting method. CoQ 10 solubility and dissolution increased with increasing concentrations of $\mathrm{P} 407$ in SDs. Comparison of the enhanced dissolution of CoQ 10 from different poloxamers suggested that the preparation of CoQ 10 SDs using P 407 as a meltable hydrophilic polymer carrier could be a promising approach to improve its dissolution.
\end{abstract}

Key words - Coenzyme Q10, Poloxamer 407, Solid dispersion, Solubility, Dissolution

Coenzyme Q 10 (CoQ 10) is a lipid soluble substance that functions as an integral part of electron transport of oxidative phosphorylation in inner mitochondrial membrane (Folkers, 1986). It is used as a nutritional supplement, antioxidant and in the treatment of cardiovascular disorders such as angina pectoris, hypertension, and congestive heart failure. It is practically insoluble in water and poorly absorbed $\left(\mathrm{T}_{\max } 5-10 \mathrm{~h}\right.$ ) from the gastrointestinal tract due to its poor dissolution (Greenberg and Fishman, 1990). Dissolution thus becomes the rate limiting step for its absorption, and the enhancement of its dissolution is desirable. Many approaches for formulating CoQ 10 have been reported. Oil based or powder filled capsules and tablet formulations are currently available on the market as nutritional supplements (Kommurur et al., 2001; Weis et al., 1994). However, CoQ 10 dissolution from these formulations differs widely and in many cases is low (Kishi et al., 1984). Other reported formulation strategies include a solubilized system with soy lecithin (Takada et al., 1985), a micellar solution of CoQ 10 with polyoxyethylene (60) hydrogenated castor oil (Kimura et al., 1986), lipid microspheres prepared as a soybean oil emulsified with yolk phospholipids (Ozawa et al., 1986), a redispersible dry emulsion (Takeuchi 1999), the complexation of CoQ 10 with cyclodextrins (Lutka and Pawlaczyk, 1995), self-emulsifying drug delivery systems (Kommurur et

\footnotetext{
Corresponding Author :

Tel : +82-53-810-2812, E-mail : csyong@yumail.ac.kr Tel : +82-31-400-5802, E-mail : hangon@hanyang.ac.kr DOI : 10.4333/KPS.2010.40.5.277
}

al., 2001), a solubilized form of CoQ 10 in a blend of polysorbate 80 and medium chain triglycerides (Chopra et al., 1998). However, not all of these strategies did greatly improved CoQ 10 solubility or dissolution, and dissolution profiles are not reported for most of these formulations either due to their oily nature and poor aqueous solubility or due to the absence of a suitable dissolution medium. Further, these approaches were tedious, time consuming and costly.

Solid dispersions (SDs) of poorly water soluble drugs in hydrophilic carrier matrix have been reported to improve their solubility and dissolution rate (Passerini et al., 2002; Seo et al., 2003; Serajuddin et al., 1999). However, CoQ 10 SDs using solvent or solvent-melting method could be problematic because, it might not be always easy to find a common solvent, large volumes of solvents and long duration of heating might be necessary to enable complete dissolution of both components, and the common methods such as vacuum drying, spray drying, spraying on sugar beads using a fluidized bedcoating system, lyophilization etc used for the removal of organic solvents from SDs could make the process relatively more complicated, tedious and costly. In addition, they might also associate with the solvent related environmental problems (Seo et al., 2003). Although, SDs by melting could be problematic (for drugs with higher melting temperature) because of the possible thermal instability of the components, and the hardening of melts resulting into difficulties in the pulverization for subsequent formulation, in case of CoQ 10 because of its low melting temperature, melting at lower temperature using meltable hydrophilic polymers might be feasible. How- 
ever, the traditional melting methods have been reported to be associated with many processing difficulties such as the temperature and shear rate control, reproducibility, scalability etc. In addition, its photo-unstability could be of particular concern while selecting the equipment, or a method. Although for many drugs, SDs by melt agglomerations in high shear mixers using a hot solution of meltable hydrophilic carriers as a binding solution have been claimed to be advantageous industrially (Passerini et al., 2002; Seo et al., 2003; Vilhelmsen et al., 2005), they were also associated with many disadvantages e.g. separate melting of polymer with or without drug was an extra step that could make the process complicated and costly, the yield in many cases was low because of the polymer/drug loss while pouring into the powder mix, and the processes themselves were very much similar to the wet granulation method used in tablet manufacturing process, thus making them relatively more demanding in terms of time and technology. In addition, the use of inert fillers such as lactose etc increased the bulk and the price of these formulations (Passerini et al., 2002; Seo et al., 2003; Vilhelmsen et al., 2005). Therefore, it would be an advantage if the formation of CoQ 10 SDs could be achieved using a rapid, less expensive, controllable and reproducible process.

Poloxamers are polyoxyethylene-polypropylene block copolymer nonionic surfactants that have been widely used as wetting and solubilizing agents, and surface adsorption excipients (Collett and Popli, 2000). They have been employed to enhance the solubility, dissolution and bioavailability of many poorly water soluble drugs using various techniques including melting agglomeration, and melting (Chen et al., 2004; Passerini et al., 2002; Rouchotas et al., 2000; Seo et al., 2003; Yu et al., 2007). For some drugs, the improvement in solubility using poloxamers was higher compared to the other meltable polymers such as polyethylene glycols or complex forming agents such as cyclodextrins (Chutimaworapan et al., 2000). In this study, various hydrophilic polymers were screened for their effect on CoQ 10 solubility and the P 407 was selected as the meltable polymers to prepare SDs using low temperature melting method because of its CoQ 10 solubility enhancing effect, low melting point, surfactant properties and oral safety (Craig, 1990; Passerini et al., 2002; Seo et al., 2003; Serajuddin et al., 1999; Vilhelmsen et al., 2005).

\section{Experimental}

\section{Materials}

CoQ 10 was supplied by Dong-A Pharm. Co. (Anyang, South Korea). Poloxamer 407 was purchased from BASF<smiles>CCC(C)(/C=C(\C)CC=O)C1=C(C)C(=O)C(OC)=C(OC)C1=O</smiles>

Figure 1. Structure of Coenzyme Q10.

Aktiengesellschaft (Ludwigshafen, Germany). Polyvinylpyrrolidone K-30 (PVPK30), hydroxyl propylcellulose and polyethylene glycol 4000 were obtained by Dong-A Pharm. Co. (Anyang, South Korea). All other chemicals were of reagent grade and used without further purification.

\section{Selection of hydrophilic the carrier polymer}

Required amount of CoQ 10 and polyvinylpyrrolidone K-30 (PVPK30), hydroxyl propyl cellulose, polyethylene glycol 4000 and P 407 (CoQ 10: Polymer 1:30 w/w ratio) were mixed for $5 \mathrm{~min}$ in a glass container to get a homogeneous mixture and sieved through a 40-mesh screen. Exactly weighed $150 \mathrm{mg}$ each of these physical mixtures was added to $10 \mathrm{~mL}$ distilled water in a screw-capped test tube, wrapped with aluminium foil, vortexed for 2 minutes and shaken in dark at $25^{\circ} \mathrm{C}$ or $37^{\circ} \mathrm{C}$ in a temperature controlled water bath (Shaking water bath KMC $12055 \mathrm{WI})$ at $150 \mathrm{rpm}$. After 24 hours, samples were filtered $(0.20 \mu \mathrm{m})$, suitably diluted with distilled water of the corresponding temperatures and analyzed by HPLC.

\section{Preparation of solid dispersions, and determination of drug content and percent yield}

CoQ 10 and P 407 in 1:1, 1:5, 1:7, 1:10, 1:15, 1:20, 1:25, and 1:30 weight ratios were separately mixed in a mortar and pestle to obtain homogeneous physical mixtures that were sieved through 40 mesh screens and transferred into a locally designed ointment formulation vessel which was wrapped with aluminium foil to prevent the photodegradation of CoQ 10 (Figure 2). Hot water $\left(65-70^{\circ} \mathrm{C}\right)$ was continuously circulated using a temperature controlled circulating water bath and the resulting molten solution was magnetically stirred at $700 \mathrm{rpm}$. After 10-15 minutes, the solution was cooled by circulating cold water $\left(<4^{\circ} \mathrm{C}\right)$ for about one hour and the solidified SDs were then ground by using a mortar and pestle, sieved through a 40 mesh screen and stored in a screw capped vial at $4^{\circ} \mathrm{C}$ until further use. Drug content was calculated by dissolving SDs equivalent to $5 \mathrm{mg}$ CoQ 10 in a suitable quantity of methanol, filtering $(0.20 \mu \mathrm{m})$, suitably diluting with methanol and analyzing by HPLC. Similarly, the percentage yield of each for- 


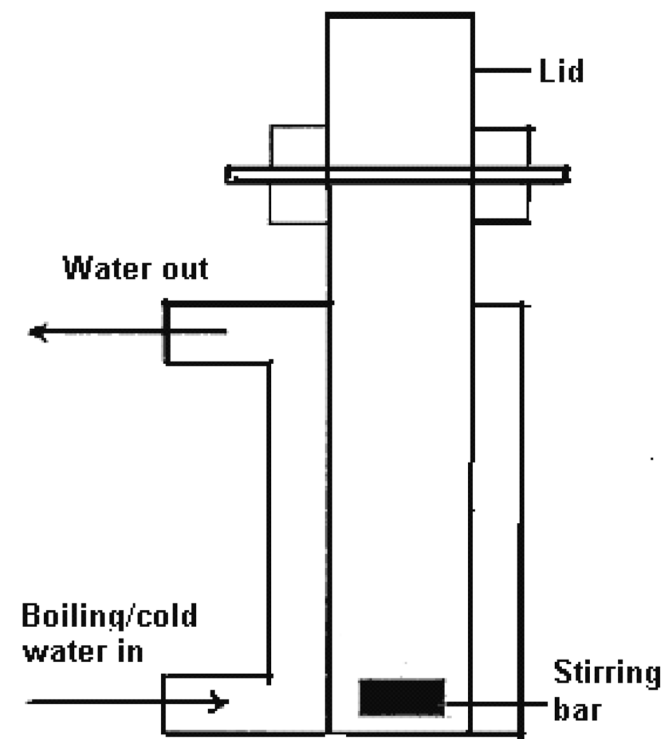

Figure 2. Locally designed micro-ointment formulation vessel.

mulation was determined according to the total recoverable final weight of SDs and the total original weights of CoQ 10 and $\mathrm{P} 407$ used.

\section{Determination of solubility}

CoQ 10, physical mixtures or SDs equivalent to $50 \mathrm{mg}$ of CoQ 10 were added to $10 \mathrm{~mL}$ distilled water in screw capped test tubes, wrapped with aluminium foil, vortexed for 2 minutes and shaken in dark at $25^{\circ} \mathrm{C}$ or $37^{\circ} \mathrm{C}$ in a temperature-controlled water-bath (Shaking water bath KMC 12055 WI). After 24 hours, resultant samples containing undissolved SDs suspended in the test medium were centrifuged at $10000 \mathrm{rpm}$ for 5 minutes and the clear supernatants obtained were filtered $(0.20 \mu \mathrm{m})$, suitably diluted with distilled water of corresponding temperatures and analyzed by HPLC.

\section{Stability of CoQ 10 during dissolution test and dissolution studies}

Nine hundred milliliter of pure CoQ 10 solution in distilled water/absolute alcohol mixture $(99: 1 \% \mathrm{v} / \mathrm{v})$ was placed in the beaker of the US Pharmacopeia (USP) model digital tablet dissolution test apparatus (Shinseang Instrument Co., South Korea) that was covered with alumunium foil, warmed to $37^{\circ} \mathrm{C}$ and rotated at the paddle speed of $50 \mathrm{rpm}$. At appropriate time intervals, small aliquot of samples were withdrawn, filtered $(0.20 \mu \mathrm{m})$, and analyzed by HPLC for remaining CoQ 10 . Considering the initial concentration of CoQ 10 as $100 \%$, the remaining percentage of CoQ 10 was determined as a function of time. Dissolution studies of CoQ 10, physical mixtures and SDs (equivalent to $10 \mathrm{mg}$ CoQ 10) were performed at the pad- dle rotation speed of $50 \mathrm{rpm}$ in $900 \mathrm{~mL}$ distilled water at $37^{\circ} \mathrm{C}$. At the specified times, $5 \mathrm{~mL}$ samples were withdrawn, filtered $(0.20 \mu \mathrm{m})$, and assayed for CoQ 10 content by HPLC. $5 \mathrm{~mL}$ fresh medium, which was warmed to $37^{\circ} \mathrm{C}$, was replaced into the dissolution medium after each sampling.

\section{Determination of coenzyme $Q \mathbf{1 0}$ solubility in polymer solutions}

$0.5,1.5,3,6,8$ and $10 \mathrm{mM}$ solutions of $\mathrm{P} 407$ were prepared in water and to $10 \mathrm{~mL}$ of each of these solutions in a screwcap test tube, $50 \mathrm{mg}$ CoQ 10 was added, screw-capped, wrapped with aluminium foil, vortexed for 2 minutes and shaken in dark at $25^{\circ} \mathrm{C}$ in a temperature controlled water bath (Shaking water bath KMC $12055 \mathrm{WI}$ ) at $150 \mathrm{rpm}$. After 48 hours, resultant samples containing undissolved CoQ 10 suspended in the test medium were centrifuged at $10000 \mathrm{rpm}$ for 5 minutes and the clear supernatants obtained were filtered $(0.20 \mu \mathrm{m})$, suitably diluted with corresponding P 407 solutions and analyzed by HPLC.

\section{Drug analysis}

The concentrations of CoQ 10 was analyzed by Jasco P987 HPLC system equipped with a Jasco UV detector (UV-975), using Borwin program. HPLC separation was performed with $50 \mu \mathrm{L}$ injection volume on a reverse-phase C 18 column (Intersil GL Science column. $5 \mu \mathrm{m}$ particle size, $4.6150 \mathrm{~mm}$ ). The mobile phase was Methanol: Ethanol (7:3 v/v), and the eluent was monitored at $275 \mathrm{~nm}$ at a constant flow rate of $1.2 \mathrm{~mL} / \mathrm{min}$ (Rousseau and Varin, 1998).

\section{Results and Discussion}

\section{Selection of hydrophilic carrier polymer}

In solubility study of CoQ 10 1:30 w/w physical mixtures with different hydrophilic polymers (Figure 3 ), the highest solubility $(18.52 \mu \mathrm{g} / \mathrm{mL})$ was observed for CoQ 10: P 407 physical mixture at $37^{\circ} \mathrm{C}$ and the lowest for CoQ 10: PVPK 30 physical mixtures $(0.25 \mu \mathrm{g} / \mathrm{mL})$ in the order of PVPK30< polyethylene glycol $4000<$ hydroxy propyl cellulose $<$ P 407 at $25^{\circ} \mathrm{C}<\mathrm{P} 407$ at $37^{\circ} \mathrm{C}$. As the sole aim of finding the solubility of CoQ 10 from its physical mixtures was to select a polymer (among various hydrophilic polymers) that have relatively higher solubility enhancing effect, the CoQ 10: Polymer ratio was empirically decided to be high enough $(1: 30 \mathrm{w} / \mathrm{w})$ because the CoQ 10 was insoluble in water and an extensive review of earlier works on this compound did not reveal sufficient reports on its solubility in presence of these polymers. Solubility at $37^{\circ} \mathrm{C}$ was determined only for the most efficient 


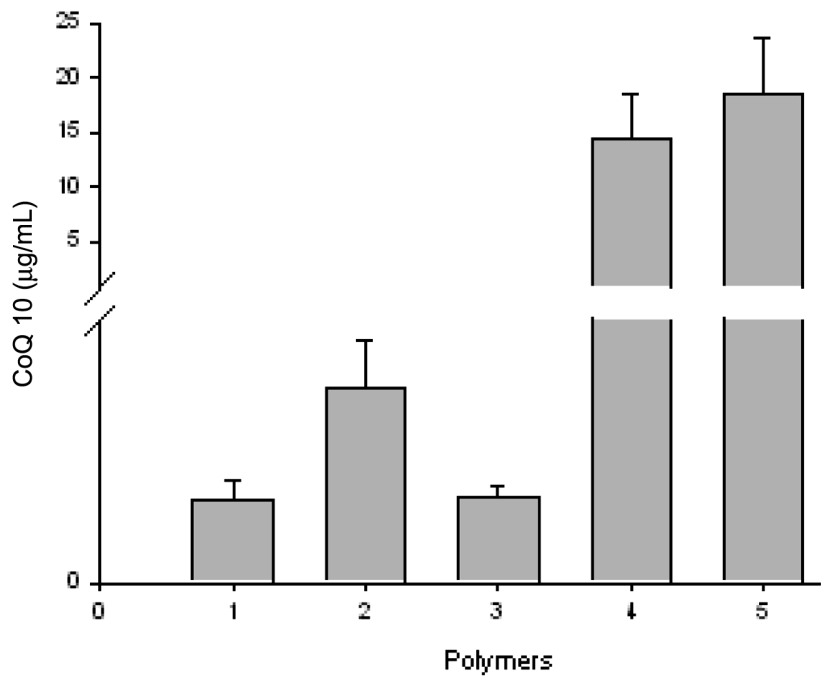

Figure 3. Solubility profiles of coenzyme Q10 from its 1:30 w/w physical mixtures with different hydrophilic polymers at $25^{\circ} \mathrm{C}$ and $37^{\circ} \mathrm{C}$. 1. Polyvinylpyrrolidone K30, 2. Hydroxypropyl Cellulose, 3 . Polyethylene Glycol 4000, 4. Poloxamer 407 at $25^{\circ} \mathrm{C}$ and 5. Poloxamer 407 at $37^{\circ} \mathrm{C}$. Data are expressed as mean $\pm \mathrm{SD}(\mathrm{n}=3)$.

polymer (CoQ 10: P 407 1: $30 \mathrm{w} / \mathrm{w}$ physical mixtures) to predict CoQ 10 solubility in physiological temperature.

Preparation of solid dispersions, and determination of drug content and percent yield

CoQ 10 assay in all SDs was almost $100 \%$ and the percentage yield was greater than $97 \%$ (data not shown). SD preparation was relatively simple and the cooled masses were easily breakable into free flowing granules of desired sizes. This could be advantageous in the large scale production of SDs. Moreover, this method was highly feasible to prepare CoQ 10- P 407 SDs because of their lower melting points. It also avoided most of the disadvantages of previously reported solid dispersion techniques in case of CoQ 10 and replaced the traditional method of melting in the frying pan, beaker etc by relatively practical apparatus for the easier control of process variables such the temperature, shearing rate etc. Another advantage was the short duration of preparation (about 1-2 hours). In addition, the results were reproducible with relatively higher percentage yields. Drug content analysis indicated that the CoQ 10 was uniformly distributed in SDs and the higher yield showed relatively lower process loss. Thus, this method could be relatively more rational approach to enhance the solubility and dissolution of CoQ 10 .

\section{Phase solubility, solubility and dissolution}

The phase solubility behavior of CoQ 10 in the presence of P 407 representing the effect of increasing the concentrations

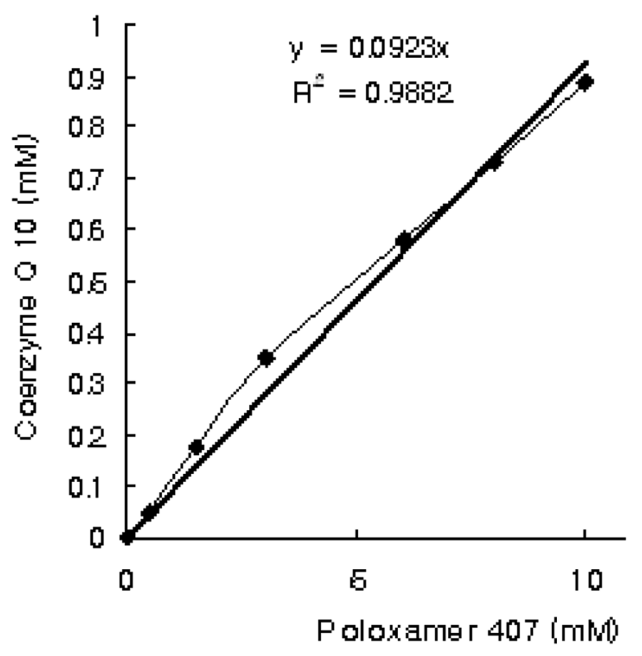

Figure 4. Phase solubility behavior of Coenzyme Q10 at $25^{\circ} \mathrm{C}$ in Poloxamer 407. Data are expressed as mean \pm SD $(n=3)$.

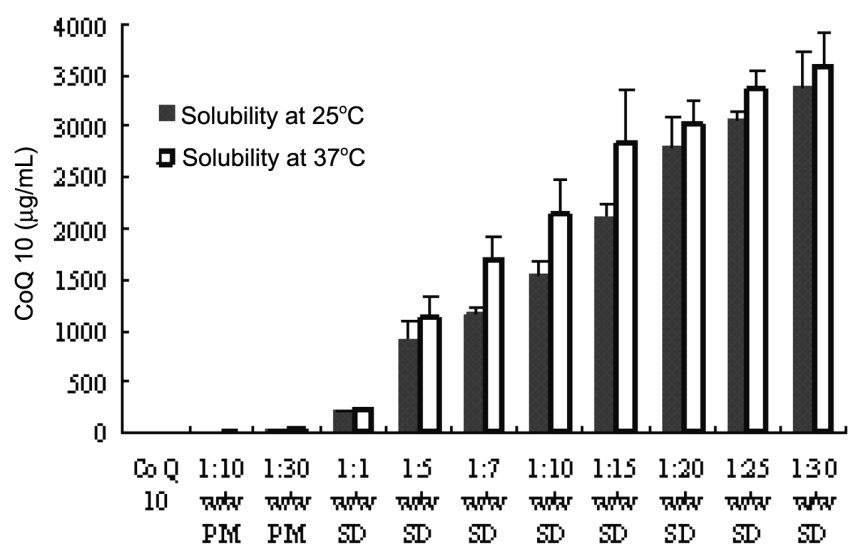

Figure 5. Solubility profile of Coenzyme Q10: Poloxamer 407 solid dispersions. Data are expressed as mean $\pm \mathrm{SD}(\mathrm{n}=3)$.

of P 407 on the apparent solubility of CoQ 10 in water at $25^{\circ} \mathrm{C}$ is presented in (Figure 4). The increase in solubility was linear $\left(\mathrm{R}^{2}=0.99\right)$ with respect to the concentration of $\mathrm{P} 407$. Solubility of CoQ 10 (Figure 5) increased with an increment in the amount of P 407 in SDs and was 6.74, 14.34, 194.96, 1524.37 and $3391.7 \mu \mathrm{g} / \mathrm{mL}$ at $25^{\circ} \mathrm{C}$, and $10.2,18.52,214.38,2145.4$ and $3582.52 \mu \mathrm{g} / \mathrm{mL}$ at $37^{\circ} \mathrm{C}$ respectively for $1: 10 \mathrm{w} / \mathrm{w}$ physical mixture, 1:30 w/w physical mixture, 1:1, 1:10 and 1:30 w/w SDs. Increase in CoQ 10 solubility with the increment of temperature might be due to the temperature aided increased in solubility, low melting point of CoQ 10, and a favorable interaction of P 407 in SDs with water at higher temperature. Any possibilities of the photolysis, thermolysis and other types of unreported degradation of CoQ 10 under the actual dissolution test conditions were determined in dissolution medium - the distilled water. The results (Figure 6) showed that CoQ 10 was stable for 18 hours and was sufficiently protected to carry dis- 


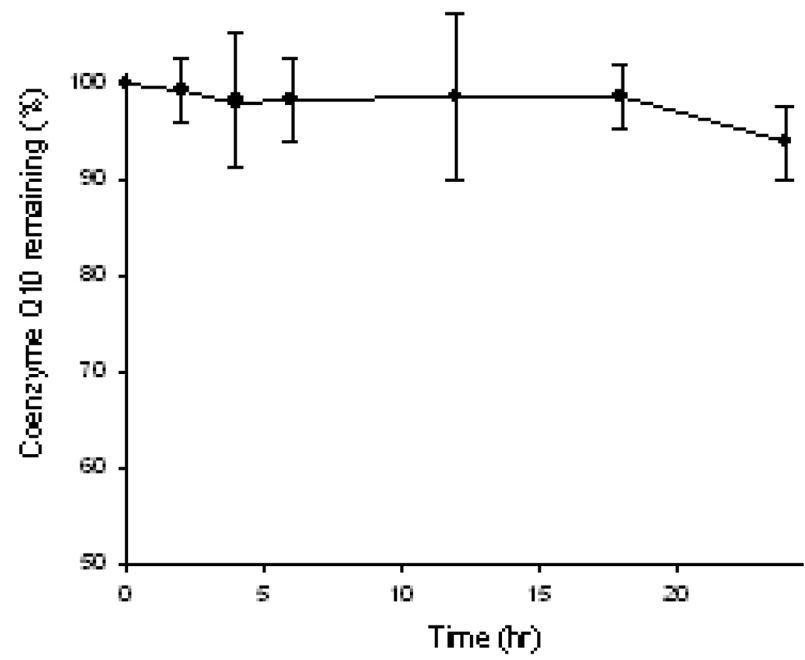

Figure 6. Stability profile coenzyme Q10 in dissolution media under actual dissolution test conditions. Data are expressed as mean $\pm \mathrm{SD}(\mathrm{n}=3)$.

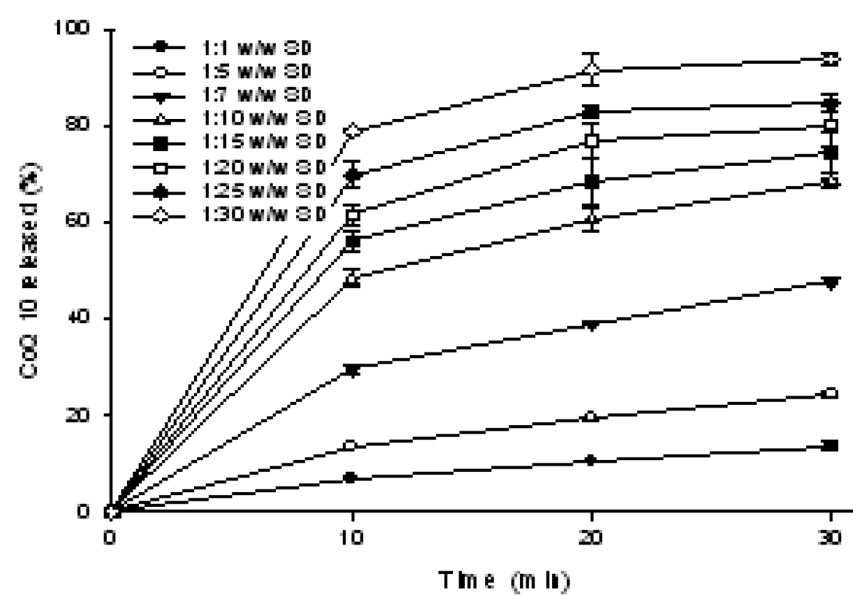

Figure 7. Dissolution profiles of coenzyme Q10: Poloxamer 407 solid dispersions water at $37^{\circ} \mathrm{C}$. Data are expressed as mean $\pm \mathrm{SD}$ $(n=3)$

solution test. $1 \%(\mathrm{v} / \mathrm{v})$ absolute alcohol was used to affect CoQ 10 solution, test was performed under yellow light and the apparatus was covered by aluminum foil to minimize any possible photodegradation. As the aim was to improve CoQ 10 dissolution rate, dissolution studies (Figure 7) were performed for initial 20 minutes. It is evident that the onset of dissolution of pure CoQ 10 and physical mixtures could not be detected in the dissolution medium till the end of the test (lower detection limit $20 \mathrm{ng} / \mathrm{mL}$ ). However, the CoQ 10 dissolution was considerably enhanced from SDs.

Most of the dissolution studies concerning CoQ 10 and other poorly water-soluble drugs have been performed in dissolution mediums that are different from those normally used for water soluble drugs e.g. incorporation of a small amount of sur- factants or acids etc in the dissolution medium. The use of exogenous surfactants in the dissolution medium may accelerate the in vitro dissolution of poorly water-soluble drugs by their wetting, micellar solubilization, and/or deflocculation properties. Hence, the dissolution of the same formulation may be very low in pure water. This problem has been previously reported by some authors. Hsu et al. (2003) could not detect CoQ 10 release over 7 days when the nanoparticles were suspended in water, presumably due to the low aqueous solubility of CoQ 10 from nanoparticles. Therefore, he used 5\% Tween 80 solution as the medium for further study. However, after 150 hours only $15 \%$ of CoQ 10 was released from nanoparticle in the dissolution media containing 5\% Tween 80 . Thus, the conclusion of increased dissolution of CoQ 10 from improved formulations cannot be justified until control dissolution in water is carried and compared.

Enhanced solubility and dissolution rate of CoQ 10 could be correlated to the chemical structure of highly water soluble $\mathrm{P}$ 407. Arrangement of ethylene oxide (EO) and propylene oxide (PO) blocks in P 407 results in an amphiphilic structure, which has the properties to self-assemble into micelles in aqueous solution (Kabanov et al., 2002). The hydrophobic core (PO block) can act as reservoir for the drug, while the hydrophilic portion (EO) acts as interface between the aqueous medium and the drug. At low concentrations, approximating those at which more conventional nonionic detergents form micelles, the poloxamer monomers are thought to form monomolecular micelles by a change in configuration in solution. At higher concentration, these monomolecular micelles associate to form aggregates of varying size, which have the ability to solubilize drugs and to increase the stability of solubilized agents (Jones and Leroux, 1999). Solubilization is likely to occur through the following mechanism. In the dry state, drug particles were in close contact or adhered to the polymer particles (shown by SEM). When they came in contact with water, the polymer particles might have hydrated rapidly into polymer solution solubilizing the adjacent drug particles and subsequently releasing the drug into the medium (Chen et al., 2004; Craig, 2002; Mura et al., 1996). This could also possibly explain the higher solubility of drug in phase solubility study where the CoQ 10 particles were already dispersed in aqueous polymer solutions. Enhanced solubility and dissolution could possibly be because of the combined action of the surface activity, solubilization and wetting effect of P 407 (Chutimaworapan et al., 2000; Passerini et al., 2006; Passerini et al., 2002; Rouchotas et al., 2000; Seo et al., 2003; Shin and Cho, 1997; Yu et al., 2007). 


\section{Conclusion}

Solubility and dissolution of CoQ 10 were remarkably improved by formulating its solid dispersions with $\mathrm{P} 407$ in a relatively simple, quick, inexpensive, reproducible and potentially scalable manner using the low melting temperature method. Preliminary results from this study suggested that the preparation CoQ 10 SDs using P 407 as a meltable hydrophilic polymer carrier could be a promising approach to its improve solubility and dissolution.

\section{Acknowledgement}

This work was supported by a grant from the Korean Health Technology R\&D Project, Ministry for Health, Welfare and Family Affairs, Republic of Korea (A092018).

\section{References}

Chen, Y., Zhang, G.G.Z., Neilly, J., Marsh, K., Mawhinney, D., Sanzgiri, Y.D., 2004. Enhancing the bioavailability of ABT - 963 using solid dispersion containing pluronic F -68, Int. J. Pharm., 286, 69-80.

Chopra, R.K., Goldman, R., Sinatra, S.T., Bhagavan, H.N., 1998. Relative bioavailability of coenzyme Q10 formulations in human subjects, Int. J. Vit. Nutr. Res., 68, 109-113.

Chutimaworapan, S., Ritthidej, G.C., Yonemochi, E., Oguchi, T., Yamamoto, K., 2000. Effect of water soluble carriers on dissolution characteristics of nifedipine solid dispersions, Drug Dev. Ind. Pharm., 26, 1141-1150.

Collett, J.H., Popli, H., 2000. Poloxamers In: A. H. Kibbe, Editor, Handbook of Pharmaceutical Excipients (3rd Edition ed), Pharmaceutical Press, London. pp. 385-388.

Craig, D.Q.M., 2002. The mechanisms of drug release from solid dispersions in water-soluble polymers, Int. J. Pharm., 231, 131-144.

Craig, D.Q.M., 1990. Polyethylene glycols and drug release, Drug Dev. Ind. Pharm., 16, 2501-2506.

Folkers, K., Wolaniuk, A., Vadhanavikit, S., Sakmato, N., Takemura, K., Baker, L., Richardson, P.C., 1986. Biomedical and clinical research on coenzyme Q10 with emphasis on cardiac patients, In: Folkers, K., Yamamura, Y. (Eds.), Biomedical and Clinical Aspects of Coenzyme Q, Vol.5. Elsevier, Amsterdam, pp. 375-391.

Greenberg, S., Fishman, W.H., 1990. Coenzyme Q10: A new drug for cardiovascular disease, J. Clin. Pharmacol., 30, 590-608.

Hsu, C.H., Cui, Z., Russell, J.M., Michael, J., 2003. Preparation and characterization of novel coenzyme $\mathrm{Q}_{10}$ nanoparticles engineered from microemulsion precursors, AAPS PharmSciTech. 4 (3), E32.

Jones, M.C., Leroux, J.C., 1999. Polymeric micelles- a new generation of colloidal drug carriers, Eur. J. Pharm. Biopharm., 48,
101-111.

Kabanov, A.V., Batrakova, E.V., Alakhov, V.Y., 2002. Pluronic block copolymers as novel polymer therapeutics for drug and gene delivery, J. Contr. Rel., 82, 189-212.

Kimura, A., Yamaguchi, H., Watanabe, K., Hayash, I.M., Awazu, S., 1986. Factors influencing the tissue distribution of cownzymq Q10 intravenously administered in an emulsion to rats; emulsifying agents and lipoprotein lipase activity, J. Pharmacol., 38, 659-662.

Kishi, H., Kanamori, N., Nishii, S., Hiraoka, E., Okamato, T., Kishi, T., 1984. In: Folkers, K., Yamamura, Y. (Eds.), Biomedical and Clinical Aspects of Coenzyme Q, Elsevier, Amsterdam, pp. 131-142.

Kommurur, T.R., Gurley, B., Khan, M.A., Reddy, I.K., 2001. Selfemulsifying drug delivery systems (SEDDS) of coenzyme $\mathrm{Q}_{10}$ : formulation development and bioavailability assessment, Int. J. Pharm., 212, 233-246.

Lutka, A., Pawlaczyk, J., 1995. Inclusion complexation of coenzyme $\mathrm{Q}_{10}$ with cyclodextrins, Acta Pol. Pharm., 52, 379-386.

Mura, P., Manderioli, A., Bramanti, G., Ceccarelli, L., 1996. Properties of solid dispersions of naproxen in various polyethylene glycols, Drug Dev. Ind. Pharm., 22, 909-916.

Ozawa, Y., Mizushima, Y., Koyama, I., 1986. Intestinal absorption enhancement of coenzyme Q10 with lipid microsphere, Drug Res., 36, 689-690.

Passerini, N., Albertini, B., Perissutti, B., Rodriguez, L., 2006. Evaluation of melt granulation and ultrasonic spray congealing as techniques to enhance the dissolution of praziquantel, Int. J. Pharm., 318, 92-102.

Passerini, N., Gonzalez-Rodriguez, M.L., Cavallari, C., Rodriguez, L., Albertini, B., 2002. Preparation and characterisation of ibuprofen-poloxamer 188 granules obtained by melt granulation, Eur. J. Pharm. Sci., 15, 71-78.

Rouchotas, C., Cassidy, O.E., Rowley, G., 2000. Comparison of surface modification and solid dispersion techniques for drug dissolution, Int. J. Pharm., 195, 1-6.

Rousseau, G., Varin, F., 1998. Determination of ubiquinone-9 and 10 levels in rat tissues and blood by high-performance liquid chromatography with ultraviolet detection, J. Chromatogr. Sci., $36,247-52$.

Seo, A., Holm, P., Kristensen, H.G., Schæfer, T., 2003. The preparation of agglomerates containing solid dispersions of diazepam by melt agglomeration in a high shear mixer, Int. J. Pharm., 259, 161-171.

Serajuddin, A.T.M., 1999. Solid dispersion of poorly water-soluble drugs: early promises, subsequent problems, and recent breakthroughs, J. Pharm. Sci., 88, 1058-1066.

Shin, S.C., Cho, C.W., 1997. Physicochemical characterizations of piroxicam-poloxamer solid dispersion, Pharm. Dev. Technol., 2, 403-40.

Takada, M., Yuzuriha, T., Katayama, K., Yamato, C., Koyama, N., 1985. Targeting of Coenzyme Q10 solubilized with soy lecithin to heart of guinea pigs, J. Nutr. Sci. Vitaminol., 31,115120. 
Takeuchi, H., Sasaki, H., Niwa, T., Hino, T., Kawashima, Y., Uesugi, K., Ozawa, H., 1992. Improvement of photostability of ubidecarenone in the formulation of a novel powdered dosage form termed redispersible dry emulsion. Int. J. Pharm., 86, 25-33.

Vilhelmsen, T., Eliasen, H., Schæfer, T., 2005. Effect of a melt agglomeration process on agglomerates containing solid dispersions, Int. J. Pharm., 303, 132-142.
Weis, M., Mortensen, S.A., Rassing, M.R., Moller, S.J., Poulsen, G., Rasmussen, S.N., 1994. Bioavailability of four oral coenzyme $\mathrm{Q}_{10}$ formulations in healthy volunteers, Mol. Aspects Med., 15(suppl): S273-280.

Yu, H., Chun, M.K., Choi, H.K., 2007. Preparation and characterization of piroxicam/poloxamer solid dispersion prepared by melting method and solvent method. J. Kor. Pharm. Sci., 37, 1-5. 